\section{Cholesterin niedrig, AIDA schadet Diabetesrisiko hoch? Frauenherzen}

In einer Auswertung mit insgesamt 50.775 Typ-2-Diabetikern plus 270.269 Kontrollen sowie 60.801 KHK-Patienten und 123.504 herzgesunden Kontrollpersonen hat sich herausgestellt, dass für Allele im NPC1L1-Gen, die mit niedrigeren LDLCholesterin-Werten assoziiert sind, ein inverser Zusammenhang mit der koronaren Herzerkrankung sowie ein direkter mit Diabetes Typ 2 besteht. So verringerte sich das KHK-Risiko um 40\%, wenn der Cholesterinwert aufgrund des genetischen Profils um $1 \mathrm{mmol} / \mathrm{l}$ abfiel, das Diabetesrisiko hingegen stieg um mehr als das Doppelte. Ähnliches gilt auch für andere cholesterinsenkenden Allele.
In einer Studie mit insgesamt 62.995 Teilnehmern war das Herzinfarktrisiko bei Typ-2-Diabetikern etwa doppelt so hoch wie bei Gesunden. Das galt für Frauen und Männer gleichermaßen. Anders bei Autoimmundiabetes (AIDA): Während im Vergleich zu gesunden Frauen AIDA-Patientinnen ein fast viermal so hohes Herzinfarktrisiko in Kauf nehmen mussten, blieb das Risiko für männliche AIDA-Patienten im Vergleich zu ihren stoffwechselgesunden Geschlechtsgenossen unverändert. Insgesamt hatten AIDA-Patienten ein deutlich besseres metabolisches Profil als Typ-2-Diabetiker, bei der glykämischen Kontrolle schnitten sie aber schlechter ab.

Laugsand LE et al. J Intern Med 2016

\section{Soziale Probleme durch Diabetes}

Diabetiker müssen ihren Alltag gezwungenermaßen ihrer Krankheit unterordnen von Ernährungsumstellung bis hin zu Insulinspritzen und Kontrollen beim Arzt greift die Krankheit meist massiv ins Leben der Betroffenen ein. Hinzu kommen soziale und ökonomische Benachteiligungen, die schon bei den kleinsten Patienten in der Schule anfangen. Was tun gegen die krankheitsbedingte Diskriminierung? Lesen Sie dazu den Schwerpunkt „Diabetes" ab S. 24!

Claudia Daniels Redakteurin

\title{
Flash-Glukose-Monitoring senkt Hypoglykämierisiko
}

\section{Diabetiker sterben früher - aber woran?}

Eine strenge Blutzuckerkontrolle bei Typ1-Diabetikern erfordert, damit es nicht zu Hypoglykämien kommt, eine engmaschige Überwachung des Glukosespiegels. Bei der Verwendung herkömmlicher Blutzuckermessgeräte müssen sich die Patienten dafür mehrmals täglich piksen. Beim Flash-Glukose-Monitoring entfällt die Blutzuckermessung, weil der Sensor vorkalibriert ist. Der Sensor, der den Glukosespiegel in der Gewebsflüssigkeit misst, wird mit einer Setzhilfe am Oberarm platziert und ver- bleibt dort für 14 Tage. Um den aktuellen Wert und die Entwicklung des Glukosespiegels in Erfahrung zu bringen, wird ein kleines Lesegerät für eine Sekunde in die Nähe des Sensors gehalten. In einer randomisierten Studie bei gut kontrollierten Typ1-Diabetikern resultierte eine Reduktion von Dauer und Zahl der Hypoglykämien, die nicht mit einer Verschlechterung der Stoffwechselkontrolle einherging.

Bolinder J et al. Lancet 2016; 388: 2254-63

\section{Metformin trotz Niereninsuffizienz prognostisch günstig}

Leiden Diabetiker zusätzlich unter chronischer Nierenkrankheit, eingeschränkter Leberfunktion oder Herzinsuffizienz, galt der Einsatz des Antidiabetikums Metformin wegen erhöhten Risikos für Laktatazidosen klassischerweise als kontraindiziert. Nun legen die Ergebnisse einer Übersichtsarbeit nahe, dass auch bei diesen Komorbiditäten die Therapie mit Metformin mit einer reduzierten Gesamtsterblichkeit assoziiert ist. Zwei Metaanalysen zufolge hatten Diabetiker mit komorbider Nieren- insuffizienz bzw. komorbider Herzinsuffizienz im Follow-Up-Zeitraum ein je $22 \%$ niedri-

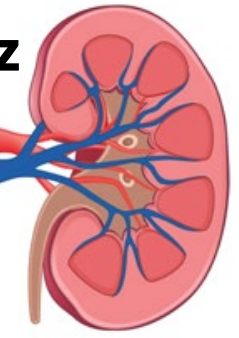
geres Mortalitätsrisiko im Vergleich zu Patienten, die kein Metformin einnahmen. In einer Studie zur Leberkomorbidität war die Einnahme von Metformin ebenfalls mit einem signifikant längeren Überleben assoziiert.

Crowley MJ et al. Ann Intern Med. 2017 (online first)
Mit der Stoffwechselerkrankung Diabetes steigt bekanntermaßen das kardiovaskuläre Risiko. Das Mehr an Herzinfarkten, Schlaganfällen und Herzversagen trägt zu der durchschnittlich kürzeren Lebenserwartung zuckerkranker Menschen bei. Inwiefern auch andere Ursachen eine Rolle spielen könnten, haben Mediziner aus Spanien evaluiert und das Sterberisiko bezogen auf verschiedene Todesursachen berechnet. Sie analysierten dafür über einen Zeitraum von zehn Jahren die Gesundheitsdaten von insgesamt 55.292 Personen. Ergebnis: Tatsächlich hatten Diabetiker nicht nur ein insgesamt höheres Sterberisiko, auch bei Betrachtung der einzelnen Todesursachen - unterteilt in kardiovaskulär, krebsbedingt sowie nichtkardiovaskulär und nichtkrebsbedingt - schnitten sie schlechter ab: Innerhalb der zehn Jahre waren 1710 Nichtdiabetiker (3,8\%) und 781 Diabetiker $(9,1 \%)$ gestorben. Das Risiko, an Herzinfarkt, Herzversagen oder Schlaganfall zu sterben, war doppelt so hoch wie das von Gesunden. Ebenfalls erhöht, wenn auch in geringerem Maße, war das krebsbedingte Sterberisiko. Gleiches galt auch für die dritte Gruppe der weder kardiovaskulären noch krebsbedingten Todesursachen.

Baena-Diez JMet al. Diabetes Care 2016:39:1987-1995 